\title{
Cellular Heterogeneity of Pancreatic Stellate Cells, Mesenchymal Stem Cells, and Cancer-Associated Fibroblasts in Pancreatic Cancer
}

\author{
Yoshiaki Sunami *D, Johanna Häußler and Jörg Kleeff $\mathbb{D}$ \\ Department of Visceral, Vascular and Endocrine Surgery, Martin-Luther-University Halle-Wittenberg, \\ University Medical Center Halle, 06120 Halle, Germany; johanna.haeussler@uk-halle.de (J.H.); \\ joerg.kleeff@uk-halle.de (J.K.) \\ * Correspondence: yoshiaki.sunami@uk-halle.de
}

Received: 10 November 2020; Accepted: 11 December 2020; Published: 15 December 2020

Simple Summary: Cancer-associated fibroblasts, which are derived from several cell types such as pancreatic stellate cells or mesenchymal stem cells, play a major role in the progression and drug resistance of pancreatic cancer. Targeting cancer-associated fibroblasts can, therefore, be a promising therapeutic strategy. However, several studies show that deletion of cancer-associated fibroblasts may also have tumor-promoting effects. These contrasting observations suggest that cancer-associated fibroblasts exhibit functional heterogeneity in pancreatic cancer.

\begin{abstract}
Pancreatic cancer is projected to become the second deadliest cancer by 2030 in the United States, and the overall five-year survival rate stands still at around $9 \%$. The stroma compartment can make up more than $90 \%$ of the pancreatic tumor mass, contributing to the hypoxic tumor microenvironment. The dense stroma with extracellular matrix proteins can be a physical and metabolic barrier reducing therapeutic efficacy. Cancer-associated fibroblasts are a source of extracellular matrix proteins. Therefore, targeting these cells, or extracellular matrix proteins, have been considered as therapeutic strategies. However, several studies show that deletion of cancer-associated fibroblasts may have tumor-promoting effects. Cancer-associated fibroblasts are derived from a variety of different cell types, such as pancreatic stellate cells and mesenchymal stem cells, and constitute a diverse cell population consisting of several functionally heterogeneous subtypes. Several subtypes of cancer-associated fibroblasts exhibit a tumor-restraining function. This review article summarizes recent findings regarding origin and functional heterogeneity of tumor-promoting as well as tumor-restraining cancer-associated fibroblasts. A better understanding of cancer-associated fibroblast heterogeneity could provide more specific and personalized therapies for pancreatic cancer patients in the future.
\end{abstract}

Keywords: pancreatic cancer; cancer-associated fibroblasts; pancreatic stellate cells; mesenchymal stem cells; cancer-restraining cancer-associated fibroblast; cellular heterogeneity

\section{Introduction}

Pancreatic ductal adenocarcinoma (PDAC) is a devastating disease with an unfavorable outcome. Currently, pancreatic cancer is the seventh leading cause of global cancer deaths in industrial countries [1], and is projected to become the second deadliest cancer by 2030 in the United States [2]. The overall five-year survival rate stands still at 9\% [3]. A number of studies have shown significant progress in survival of pancreatic cancer patients by combination chemotherapies [4-8]. However, pancreatic cancer still exhibits remarkable resistance to radiotherapy and chemotherapy, due to genetic and epigenetic alterations, together with a complex and desmoplastic tumor microenvironment $[9,10]$. 
The tumor microenvironment includes immune cells, cancer-associated fibroblasts (CAFs), which are, in part, derived from pancreatic stellate cells (PSCs) and endothelial cells, resulting in an abundant collection of inflammatory cytokines and chemokines, growth factors, and components of the extracellular matrix (ECM). The stromal compartments can make up more than $90 \%$ of the pancreatic tumor mass, which generates the hypoxic tumor microenvironment [9]. ECM proteins are produced by activated PSCs and CAFs, which support pancreatic fibrogenesis and hypoxia [11]. The dense stroma with ECM proteins can be a physical and metabolic barrier reducing therapeutic efficacy [12]. The hypoxic microenvironment leads to activation of angiogenesis, metastasis, and metabolic reprogramming, where hypoxia inducible factors (HIFs) play a central role [12]. To that end, targeting CAFs/activated PSCs or disrupting ECM are promising therapeutic strategies. The data have, however, suggested that deletion of CAFs also have tumor-promoting effects. Deletion of alpha-smooth muscle actin ( $\alpha$-SMA, a marker of myofibroblasts)-positive cells in a pancreatic cancer mouse model (Ptf1-Cre; lox-stop-lox-Kras $\left.{ }^{G 12 D /+} ; \mathrm{Tgfbr}^{l o x} / \mathrm{lox}\right)$ leads to invasive, undifferentiated tumors that are more hypoxic and reduces animal survival [13]. Deletion of $\alpha$-SMA-positive cells also diminishes animal survival in another mouse model called KPC (Pdx1-Cre; lox-stop-lox-Kras G12D/+ ; lox-stop-lox-Trp53 ${ }^{R 172 H /+}$ ) [13]. KPC and Ptf1-Cre; lox-stop-lox-Kras ${ }^{\mathrm{G} 12 \mathrm{D} /+} ; \mathrm{Tgfbr}^{\text {lox/lox }}$ are mouse models that spontaneously develop pancreatic cancer recapitulating clinical and histopathological features of the human disease $[13,14]$. Myofibroblast-depleted tumors display increased invasion associated with intra-tumoral hypoxia in Ptf1-Cre; lox-stop-lox-Kras ${ }^{\mathrm{G} 12 \mathrm{D} /+} ; \mathrm{Tgfbr}^{\text {lox/lox }}$ mice [13]. Sonic hedgehog (Shh) signaling is known to drive formation of a fibroblast-rich desmoplastic stroma [15,16]. Inhibition of Shh signaling with IPI-926, which is a semisynthetic derivative of cyclopamine, enhances delivery of gemcitabine in KPC mice [17]. However, conditional deletion of Shh $\left(S h h^{l o x} /\right.$ lox $)$ reduces stromal content, but tumors are more aggressive, undifferentiated, and display increased vascularity in the Pdx1-Cre; lox-stop-lox-Kras G12D; Trp53lox/+ pancreatic cancer mouse model [18]. Furthermore, a clinical study (NCT01130142) that combined chemotherapy with IPI-926 and gemcitabine showed a shortened patient overall survival than gemcitabine treatment alone and was, therefore, terminated prematurely [19]. These studies imply that neither removal of cells based solely on $\alpha$-SMA positivity nor abrogation of Shh expression or its pharmacologic inhibition are effective in limiting tumor aggressiveness. The fact that these treatments limit stromal development imply a restrictive role of some kinds of CAF without proving the absence of a tumor-promoting role in other kinds.

\section{Origin and Functional Heterogeneity of Cancer-Associated Fibroblasts in Cancer}

In general, activated fibroblastic cells in the tumor microenvironment of solid cancers that have a phenotype, function, or location distinct from quiescent fibroblasts are considered as CAFs [20]. CAFs are characterized by their diverse origins. Resident fibroblasts and especially PSCs are major sources of CAFs in pancreatic cancer [21-23]. Furthermore, adipocytes, pericytes, monocytes, endothelial cells, and bone marrow-derived or adipose-derived mesenchymal stem cells (MSCs) can differentiate into CAFs (Figure 1) [24-27]. Cultured MSCs themselves exhibit a high cellular plasticity and capacity to undergo osteogenic, adipogenic, myogenic, and chondrogenic differentiation [28].

In the healthy pancreas, PSCs are in the quiescent state. The most consistent marker of quiescent PSCs is the presence of cytoplasmic vitamin A droplets [22]. In addition, PSCs can be identified by the expression of desmin, glial fibrillary acidic protein (GFAP), and acetylcholine receptors (Table 1) [20]. Extracellular signals, such as transforming growth factor (TGF)- $\beta$, tumor necrosis factor (TNF)- $\alpha$, interleukins, and oxidative stress modulate PSC activation (Table 2) [22]. Besides ECM proteins, activated and transformed PSCs produce several stimulators such as platelet-derived growth factor (PDGF), connective tissue growth factor (CTGF), and epidermal growth factor (EGF), supporting cancer cell proliferation [22]. PSCs can undergo autophagy to support pancreatic fibrosis and tumor growth [29]. Autophagy of PSCs is associated with shorter survival of pancreatic cancer patients [29]. Pancreatic cancer cells stimulate autophagy in PSCs [30] and the alanine released from PSCs undergoing autophagy is utilized by pancreatic cancer cells [30]. It was discovered in the 
inducible oncogenic KRAS (Kirsten Rat Sarcoma) mouse model (Ptf1-Cre; Rosa26-rtTa; TetO-Kras G12D) in which pancreatic tumor cells regulate PSCs non-cell-autonomously by secreting factors including SHH protein [31]. Pancreatic cancer cells are further modulated by signal transduction of reciprocal signals from activated PSCs. For example, signals from SHH-activated PSCs are transduced through the insulin growth factor 1 receptor (IGF1R) on tumor cells (Table 2) [31].

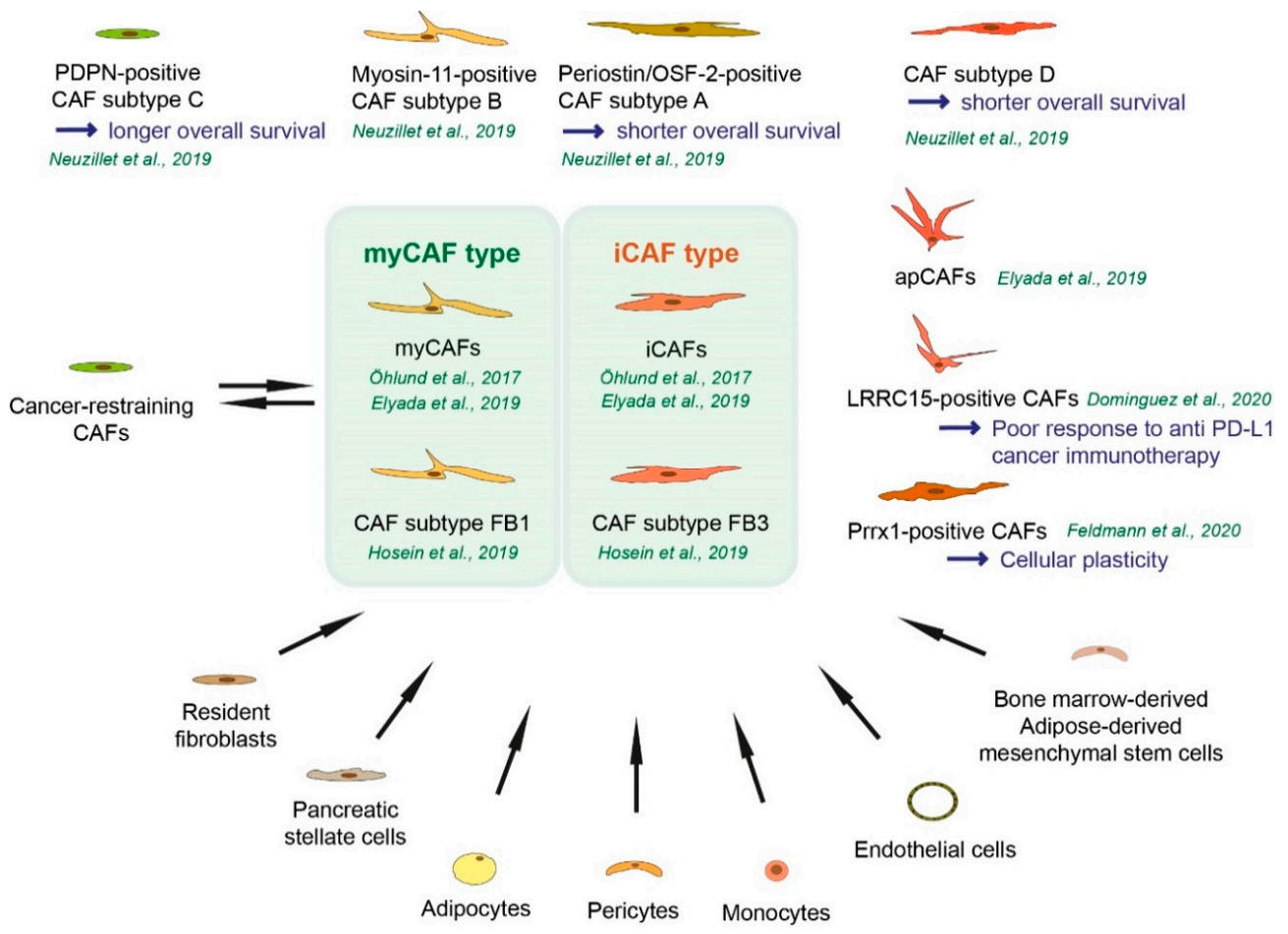

Figure 1. Origin and functional heterogeneity and classification of cancer-associated fibroblasts. Non-fibroblast lineage cells such as monocytes or endothelial cells can become a part of cancer-associated fibroblasts (CAFs). CAFs represent very heterogeneous subpopulations with different functions in tumor-promoting and tumor-restraining manner. Tumor-promoting CAFs can be converted to tumor-restraining CAFs and vice versa.

Table 1. Origin of cancer-associated fibroblasts (CAFs) and their markers.

\begin{tabular}{ccc}
\hline Cell Type & Selected Markers/Factors Mentioned in the References & Reference \\
\hline $\begin{array}{c}\text { Pesenchymal stem cell-derived cancer-associated } \\
\text { fibroblasts (Gastric cancer) }\end{array}$ & Desmin, GFAP, acetylcholine receptors & {$[20]$} \\
\hline $\begin{array}{c}\text { Bone-marrow derived mesenchymal stem cells } \\
\text { (Breast cancer) }\end{array}$ & IL-6, Wnt5a, BMP4 & {$[32]$} \\
\hline $\begin{array}{c}\text { Cancer-associated mesenchymal stem cells } \\
\text { (Pancreatic cancer) }\end{array}$ & CCL5/RANTES & {$[33]$} \\
\hline $\begin{array}{c}\text { Adipose-derived mesenchymal stem cells } \\
\text { (obese donors) (ovarian cancer) }\end{array}$ & ACTA2, VEGF, FAP, S100A4 (FSP), CSPG4 (NG2) & {$[34]$} \\
\hline
\end{tabular}


Table 2. Soluble factors and function.

\begin{tabular}{|c|c|c|}
\hline Soluble Factors & Function & Reference \\
\hline TGF- $\beta$, TNF $\alpha$, interleukins & Pancreatic stellate cell (PSC) activation & [22] \\
\hline PDGF, CTGF, EGF & Secreted by activation and transformed PSCs for cancer cell proliferation & [22] \\
\hline $\mathrm{SHH}$ & Produced by pancreatic cancer cells for PSC regulation & [31] \\
\hline IGF1 & $\begin{array}{l}\text { Reciprocal signal from SHH-activated PSCs for pancreatic } \\
\text { cancer modulation }\end{array}$ & [31] \\
\hline IL-6, Wnt5a, BMP4 & $\begin{array}{l}\text { Produced by mesenchymal stem cell (MSC)-derived cancer-associated } \\
\text { fibroblasts (CAFs) and MSCs are recruited to the dysplastic stomach }\end{array}$ & [32] \\
\hline CCL5/RANTES & $\begin{array}{l}\text { Secreted by bone marrow-derived MSCs (BM-MSCs) to enhance breast } \\
\text { cancer motility, invasion, and lung metastasis }\end{array}$ & [33] \\
\hline GM-CSF & $\begin{array}{l}\text { Secreted by cancer-associated MSCs (CA-MSCs) for pancreatic cancer } \\
\text { invasion and metastasis }\end{array}$ & [34] \\
\hline IL-6, IL-11, LIF & $\begin{array}{l}\text { Secreted by inflammatory CAFs (iCAFs) and activate STAT3 signaling } \\
\text { in pancreatic cancer organoids }\end{array}$ & [36] \\
\hline IL-1 & $\begin{array}{l}\text { Secreted by pancreatic cancer cells for inducing LIF and JAK/STAT } \\
\text { signaling in iCAFs }\end{array}$ & [37] \\
\hline
\end{tabular}

It has been shown that, in an inflammation-induced gastric cancer model with Helicobacter felis infection, at least $20 \%$ of CAFs originate from bone marrow and derive from MSCs. MSC-derived CAFs that are recruited to the dysplastic stomach express IL-6, Wnt5a, and bone morphogenetic factor 4 (BMP4) (Tables 1 and 2) [32]. In the case of breast cancer, bone marrow-derived MSCs (BM-MSCs) cause the cancer cells to increase their metastatic potency by stimulating de novo secretion of CCL5 (also called RANTES) secreted by BM-MSCs. CCL5 acts as a reciprocal signal to the breast cancer cells to enhance their motility, invasion, and lung metastasis (Table 2) [33]. In another study, it has been shown that BM-MSCs are recruited to primary breast cancer as well as to lung metastasis. BM-MSC-derived CAFs (BM-CAFs) are a substantial source of CAFs in the tumor microenvironment. They support tumor growth and enhance angiogenesis via Clusterin expression. Resident CAFs do, but BM-derived CAFs do not express PDGFR $\alpha$. Therefore, the recruitment of BM-CAFs results in a decrease in the ratio of PDGFR $\alpha$-expressing CAFs. A decrease in PDGFR $\alpha$ expression in breast cancer patients is associated with shorter overall survival [38]. CD $44^{+} / \mathrm{CD}^{+} / \mathrm{CD} 90^{+} / \mathrm{CD} 49 \alpha^{+} \mathrm{MSC}$ are detected in pancreatic cancer and termed cancer-associated MSCs (CA-MSCs) [34]. Pancreatic CA-MSCs, but not non-MSC CAFs, promote tumor cell growth, invasion, and metastasis [34]. CA-MSCs secrete granulocyte-macrophage colony-stimulating factor (GM-CSF), and pancreatic cancer cells express GM-CSF receptor. CA-MSC-derived GM-CSF is required for pancreatic cancer cell invasion and metastasis (Table 2) [34]. The therapeutic potential of MSCs has been studied in several clinical trials including studies targeting gastrointestinal, lung, and ovarian cancer [39]. The TREAT-ME1 study (NCT02008539) used bone marrow-derived MSC-delivery of Herpes simplex virus type 1 thymidine kinase (HSV-TK) under the control of the CCL5-promotor. After cell delivery, a cytotoxic drug ganciclovir is administered, which is phosphorylated and activated by HSV-TK for cancer cell death [40]. A Phase 1 study (NCT02530047) to test the safety of interferon $\beta$ (IFN- $\beta$ )-secreting MSCs has been completed. A Phase 1/2 study (NCT03298763, TACTICAL trial) recruits lung cancer patients using MSCs to deliver the TNF-related apoptosis-inducing ligand (TRAIL). An inhibitory effect of fibroblasts on cancer cell proliferation has been proposed and named cancer-restraining CAFs [41], which is further discussed below (see Section 5). There is evidence to suggest that CAFs can arise from adipocytes and adipose-derived MSCs in several solid tumors. In the case of breast cancer, adipocyte cells co-cultured with cancer cells are differentiated into fibroblast-specific protein 1 (FSP-1)-positive, but not $\alpha$-SMA-positive, CAFs [42]. Adipose-derived MSCs are converted into CAFs in the presence of breast cancer cell-derived factors. Adipose-derived MSCs (AD-MSCs) from obese donors express higher levels of $A C T A 2$ (coding $\alpha$-SMA), VEGF, fibroblast activation protein (FAP), S100A4 (FSP), and chondroitin surface proteoglycan 4 CSPG4 (also known as NG2) when compared 
to AD-MSCs from lean donors [35]. Ovarian cancer cells induce differentiation of AD-MSCs into $\alpha$-SMA and FAP-positive CAFs [43]. AD-MSCs can differentiate into distinct CAF subtypes dependent on different co-culture systems [26]. Distinct CAF subtypes are discussed in Section 4. CAFs are characterized by their diverse origins and differentiation into distinct CAF subtypes, which depends on the proximity to other cell types.

\section{Fibroblast and Cancer-Associated Fibroblast Markers}

In a healthy pancreas, distinct fibroblast populations and mesenchymal heterogeneity are observed [44]. Two different populations of pancreatic fibroblasts have been characterized, by the expression of transcription factors GLI1 and HOXB6 [45]. The transcription factor GLI1 is a terminal effector of the Hedgehog pathway. Hedgehog signaling is active during embryonic development but largely suppressed in the adult [46]. Yet, a small population of GLI-positive fibroblasts can be observed in the healthy pancreas [47]. Activation of GLI transcription factors is required for formation of oncogenic KRAS-dependent pancreatic cancer in mice [48]. The study used Ptf1-Cre; lox-stop-lox-Kras G12D; Rosa26-Gli3T mice, where GLI3 acts as a dominant repressor of Gli transcription [48]. Furthermore, GLI1 accelerates KRAS-initiated pancreatic tumorigenesis in global Gli1 knock-in mice (Ptf1-Cre; lox-stop-lox-Kras ${ }^{G 12 D /+}$; Rosa26-Gli1) [48]. HOX6 paralogs (HOXA6, HOXB6, and HOXC6) are expressed in the mesoderm of the developing pancreas and mesenchyme but not in the endoderm [49]. GLI1 and HOXB6 are expressed in largely distinct populations in the healthy pancreas. Lineage-tracing experiments revealed that GLI1-positive cells, but not Hoxb6-positive cells, which proliferated and promoted the fibrotic reaction in pancreatic cancer. In a pancreatic cancer mouse model (Ptf1-Cre; frp-stop-frp-Kras ${ }^{G 12 D /+}$; Gli1 ${ }^{C r e E R T}$ ), lineage-traced, GLI1-positive cells account for a little less than half of the total myofibroblast population [45].

In response to tissue injury or stimuli, quiescent fibroblasts are activated to become a normal activated fibroblast (NAFs) to facilitate repair and regeneration. The NAFs gain expression of $\alpha$-SMA and vimentin. The NAFs further gain contractile properties along with enhanced ECM production, remodeling, and cytoskeletal rearrangement. With persisting injury or with the development of cancer lesions, activated fibroblasts further gain enhanced proliferation properties and form a functionally diverse population as fibrosis-associated fibroblasts (FAFs) or CAFs, respectively [28].

A number of different markers can identify activated fibroblasts including $\alpha$-SMA, desmin, FAP, fibroblast-specific protein (FSP1, also known as S100A4), PDGFR $\alpha$, PDGFR $\beta$, podoplanin (PDPN), and vimentin $[20,28]$. However, proposed activated fibroblast markers are not specific for fibroblasts or activated fibroblasts [28]. For example, FSP1 has been identified in an inflammatory subpopulation of macrophages in the liver [50]. FAP is widely considered one of the most reliable CAF markers. However, only a certain subpopulation of CAFs expresses FAP and it is completely absent in other tumor fibroblast subpopulations [51]. A Phase 2 trial of FAP inhibition using the humanized monoclonal antibody sibrotuzumab failed to slow tumor progression in colorectal cancer patients (NCT02198274) $[10,52]$. $\alpha$-SMA has been considered as a key marker for identifying CAF populations. High expression of $\alpha$-SMA in CAFs is associated with shorter overall survival in breast and colon cancer $[53,54]$. However, the ACTA2 gene (coding $\alpha$-SMA) expression in patients-derived CAFs and Acta2 gene and $\alpha$-SMA expression in murine (KPC mice)-derived CAFs or primary PSCs drop when cultured with conditioned media from organoids derived from pancreatic cancer patients or from organoids derived from KPC mice, respectively [36] (see Section 4.). Therefore, $\alpha$-SMA cannot be used for all CAF populations. PDGFRs are expressed in several cancer types, fibroblasts, astrocytes, neuro-progenitors, and pericytes $[55,56]$, but are more broadly expressed in fibroblasts than comparative markers like $\alpha$-SMA [56]. PDPN is an O-glycosylated transmembrane glycoprotein that is expressed not only in CAFs but also observed in lymphatic endothelial cells, kidney podocytes, keratinocytes, mesothelial cells, and in several types of cancer. PDPN expression in CAFs is correlated with shorter overall survival of pancreatic cancer patients [57]. Vimentin is an intermediate filament protein involved in 
the formation of the cytoskeleton network [56]. Vimentin is highly expressed in fibroblasts, but also in smooth muscle cells, endothelial cells, and cancer cells [58].

As a marker of activated fibroblasts, Y397 phosphorylated focal adhesion kinase (FAK) has been considered. FAK is a key intracellular effector of ECM signaling, which gets activated upon ECM-induced integrin receptor activation. This leads to auto-phosphorylation of FAK at Y397 [59]. CAFs from pancreatic cancer patients exhibit an increase of phosphorylated Y397 FAK compared to controls. Higher levels of Y397-phosphorylated FAK are associated with shorter disease-free and overall survival of pancreatic cancer patients [60]. Since most markers are shared with other cell types, further identification of CAF subtype-specific markers are required. Furthermore, it is possible that a single marker identifies a range of distinct CAF subtypes that may have functionally opposing roles in cancer progression [58]. Therefore, it will be necessary to subdivide CAFs by the CAF subtype-specific markers or a combination of several markers.

\section{Cancer-Associated Fibroblast Subtype Classification in Pancreatic Cancer}

To characterize the heterogeneity of fibroblasts and to understand the molecular mechanism in pancreatic cancer immunity and progression, it has been important to investigate cell type-specific gene expression. It has been shown that the majority of fibroblasts in human pancreatic tumors as well as in tumors from KPC mice express FAP and low levels of $\alpha$-SMA, whereas a subpopulation of FAP-positive cells exhibit elevated $\alpha$-SMA expression (named as myo-fibroblastic CAFs "myCAFs") [36]. CAFs co-cultured with organoids derived from pancreatic cancer patients secrete IL-6, IL-11, and leukemia inhibitory factor (LIF), and these ligands activate a signal transducer and activator of transcription 3 (STAT3) in organoids (Table 2) [36]. This cytokine-expressing CAF subpopulation with low $\alpha$-SMA expression is named inflammatory CAFs (iCAFs) [36]. In pancreatic cancer, in patients and in KPC mice, myCAFs and iCAFs co-exist [36]. MyCAFs are located in the peri-glandular region, iCAFs are located more distantly from tumor cells and myCAFs in pancreatic cancer [36]. A cluster of genes including Acta2 (coding $\alpha$-SMA), Vim, Ctgf, Col1a1, Col5a1, and Col6a1 are up-regulated in myCAFs, whereas interleukins Il1, Il6, Il11, and Lif as well as chemokines such as Cxcl1, Cxcl2 are up-regulated in iCAFs (Table 3) (Figure 1) [36]. For the transcriptomic analysis, mouse quiescent PSCs, $\alpha$-SMA ${ }^{\text {low }}$ IL- $6^{\text {high }}$ mouse PSCs cultured in trans-well with KPC tumor organoids for iCAFs, and $\alpha$-SMA ${ }^{\text {high }}$ IL-6 $6^{\text {low }}$ PSCs for myCAFs, were used [36]. AD-MSCs (see Section 2) can differentiate into distinct $C A F$ subtypes dependent on different co-culture systems, between direct contact co-culture with pancreatic cancer cells (cancer cells and AD-MSCs) are mixed or indirect contact with pancreatic cancer cells (cancer cells and AD-MSCs are separated by a trans-well membrane). Direct contact co-culture induces differentiation into myCAFs and iCAFs, while indirect co-culture induces differentiation into only iCAFs [26]. PSCs can differentiate into myCAFs and iCAFs when co-cultured with organoids derived from KPC mice [36]. These findings suggest that direct juxacrine interactions with cancer cells are required for myCAF formation [36]. 
Table 3. Cancer-associated fibroblast (CAF) subtypes and CAF-subtype-defined markers.

\begin{tabular}{|c|c|c|c|}
\hline CAF Subtypes & Markers and Factors Expressed in CAF Subtype & Reference & Additional Information \\
\hline Myofibroblastic CAFs (myCAFs) & Acta2, Vim, Ctgf, Col1a1, Col5a1, Col6a1 & [36] & \\
\hline Myofibroblastic CAFs (myCAFs) & Tnc, Tgfb1, Thy1, Tagln, Col12a1, Pdgfrb & {$[61]$} & \\
\hline Inflammatory CAFs (iCAFs) & Il1, Il6, Il11, Lif & [36] & \\
\hline Inflammatory CAFs (iCAFs) & Clec3b, Col14a1, Gsn, Ly6c1, Cxcl12 & [61] & \\
\hline CAF subtype A & Periostin/OSF-2 & [23] & Shorter overall survival \\
\hline CAF subtype B & Myosin-11 & [23] & Myogenic properties \\
\hline CAF subtype $C$ & PDPN & [23] & Prolonged overall survival \\
\hline CAF subtype FB1 & $\begin{array}{c}\text { Cxcl14, Ptn, Igf1, Igfbp4, Igfbp7, Il6, Ccl2, Ccl7, } \\
\text { Cxcl12, Pdgfra }\end{array}$ & [62] & Overlap with iCAFs \\
\hline CAF subtype FB2 & Ly6a, Ly6c1, Nov, Pi16 & [62] & \\
\hline CAF subtype FB3 & $\begin{array}{c}\text { Lrrn4, Gpm6a, Nkain4, Lgals7, Msln, Cav1, Cdh11, } \\
\text { Gas6 }\end{array}$ & [62] & Overlap with myCAFs \\
\hline Antigen-presenting CAFs (apCAFs) & Slpi, Saa3, Cd74, H2-Ab1, NKain4, Irf5 & [61] & \\
\hline LRRC15-positive CAFs & LRRC15 & [63] & $\begin{array}{l}\text { Poor response to anti-PD-L1 cancer } \\
\text { immunotherapy }\end{array}$ \\
\hline Prrx1-positive CAFs & Prrx1 & [64] & Involved in CAF plasticity \\
\hline
\end{tabular}


In another study (single cell RNA sequencing) with surgically resected low-grade intraductal papillary mucinous neoplasms (IPMNs), high-grade IPMNs, and invasive pancreatic cancer, myCAFs and iCAFs are observed. The iCAF subpopulation is detected in invasive pancreatic cancer, and iCAFs exhibit elevated expression of VIM, FAP, COL3A1, DES, IL6, and CXCL12 and reduced expression of ACTA2 (coding $\alpha$-SMA). The myCAF population is rare in low-grade IPMNs but is highly represented in high-grade IPMNs, and exhibit increased expression of ACTA2 and reduced expression of CXCL12 and DES [65]. Neuzillet and colleagues performed RNA sequencing and identified four different subtypes in primary isolated CAFs from pancreatic cancer patient specimens (named subtype A to D) (Figure 1). Periostin is considered as a subtype A biomarker and high expression of periostin is associated with shorter overall survival of pancreatic cancer patients. Subtypes B-D CAFs induce more cancer cell proliferation than subtype A CAFs [23]. Periostin, or osteoblast-specific factor 2 (OSF-2), is considered an important molecule in several diseases such as scar formation in myocardial infarction, fibrosis, or cancer cell migration [22,66]. Subtype B displays myogenic properties and myosin-11, which is a smooth muscle myosin, is selected as a subtype B biomarker [23]. Subtype C CAF-related marker is PDPN, which is a cell-surface mucin-like glycoprotein. Pancreatic cancer patients with the dominant subtype C CAFs show prolonged overall survival [23]. In another study, PDPN expression in CAFs correlated with shorter overall survival of pancreatic cancer patients [57] and a study suggests heterogeneity within the PDPN-positive stroma [63]. Patients with dominant subtype D CAFs show shorter overall survival [23]. In subtype D CAFs, CXADR (Coxachie virus and Adenovirus Receptor, also known as CAR), MEOX (Mesenchyme Homeobox), and PLS1 are highly expressed [23]. CXADR is an essential regulator of cell growth and adhesion during development [67]. The MEOX family of transcription factors are important during mammalian embryogenesis and axial skeleton development [68], PLS1 (Plastin 1), also called fimbrin, is one of the most abundant actin-bundling proteins [69]. CXADR and MEOX are expressed in tumor cells, PLS1, is expressed in endothelial cells [23].

In addition to KRAS mutation, additional driver events are required for pancreatic cancer progression, where TP53 and CDKN2A are the most commonly mutated genes [70]. Ink4a/p16 inhibits cell cycle progression through the G1/S checkpoint mediated by CDK4 and CDK6 [71]. To profile cell heterogeneity during different stages of pancreatic cancer progression in Ptf1-Cre; lox-stop-lox-Kras G12D/+ ; Ink $4 a^{l o x / l o x}$ mouse model, the single-cell RNA-sequencing has been conducted [62]. In this study, there are three distinct molecular subtypes of fibroblasts in the healthy mouse pancreas, and two distinct populations of CAFs in advanced pancreatic cancer [62]. Distinct subtypes of CAFs are observed in another pancreatic cancer mouse model, Ptf1-Cre; lox-stop-lox-Kras G12D; lox-stop-lox-Trp53 ${ }^{\text {R172H/+ }}$ [62]. The two CAF subpopulations exist across different advanced-stage pancreatic cancer mouse models, suggesting a consistent cell of origin [62]. A fibroblast subpopulation in healthy mouse pancreas in Ptf1-Cre; lox-stop-lox-Kras ${ }^{G 12 D /+}$; Ink $4 a^{l o x / l o x}$ mice express chemokine Cxcl14, Ptn, and several genes associated with insulin-like growth factor (IGF) signalling such as Igf1, IGF-binding protein (IGFBP) family $I g f b p 4$, and Igfbp7 (called here FB1) [62]. The second subpopulation (FB2) express $L y 6 a, L y 6 c 1$, which is a CCN family secreted matricellular protein member Nov, and Pi16. The third population (FB3) expresses several mesothelial markers such as Lrrn4, Gpm6a, Nkain4, Lgals7, and Msln as well as Cav1, Cdh11, and Gas6 (Table 3) [62]. In early lesions, the FB2 subpopulation starts to move toward an FB1 expression profile. Il6, Ccl2, Ccl7, Cxcl12, and Pgdfra are expressed in FB1 subtype fibroblasts in the normal pancreas, as well as during the progression of pancreatic cancer [62]. The myofibroblast markers such as Acta2 and Tagln are expressed in the FB3 subpopulation. These findings can support the presence of iCAFs (FB1) and myCAFs (FB3) in the pancreatic cancer mouse models (Figure 1) [62].

The FB3 fibroblast subpopulation expresses several major histocompatibility complex (MHC) class II-associated genes [62]. In another single-cell analysis, a subpopulation of CAFs that express MHC class II and CD74 is described and named antigen-presenting CAFs (apCAFs) [61]. It is not clear whether the FB3 CAF subpopulation can be divided into myCAFs and apCAFs. The apCAF subpopulation further expresses Saa3 and Slpi, and the subpopulation detected in tumors of KPC 
mice as well as in pancreatic cancer patients. MHCII molecules of apCAFs have the capacity to present antigens to $\mathrm{CD}^{+} \mathrm{T}$ cells, but apCAFs lack the costimulatory molecules needed to induce T-cell proliferation, potentially contributing to immune suppression [61]. In this study, selected markers for each CAF subpopulation are summarized as follows: markers for myCAFs are Tnc, Tgfb1, Thy1, Tagln, Col12a1, and Pdgfrb. Selected markers for iCAF are Clec3b, Col14a1, Gsn, Ly6c1, and Cxcl12. Markers for apCAFs are Slpi, Saa3, Cd74, H2-Ab1, Nkain4, and Irf5 (Table 3) [61]. Mechanistically, IL-1 induces LIF and activates JAK/STAT signaling to generate iCAFs (Table 2) [37]. TGF $\beta$ downregulates IL1R1 and promotes differentiation into myofibroblasts [37].

A recent study identified a leucine-rich repeat containing 15 (LRRC15)-positive CAF subpopulation within the PDPN-positive CAF population in pancreatic cancer mouse model (Pdx1-Cre; lox-stop-lox-Kras ${ }^{G 12 D /+} ;$ p16/p19lox/lox $)$ [63]. The LRRC15-positive CAF subpopulation can be identified in pancreatic cancer patient specimens [63]. Mouse tumor spheroids cultured with LRRC-positive CAFs grow larger than those in media alone, suggesting that LRRC-positive CAFs can directly enhance tumor growth [63]. Elevated LRRC15 levels correlates with a poor response to anti-PD-L1 cancer immunotherapy in six immune-excluded cancer types (characterized by a dense stromal structure surrounding the tumor and the presence of most tumor-infiltrating cells in the stromal area, fewer in the tumor parenchyma), pancreatic cancer, bladder cancer, renal cell carcinoma, head and neck cancer, and non-small cell lung cancer (Figure 1) [63].

The paired-related homeobox (Prrx1) is considered a driver of cellular plasticity during pancreatic ductal development, acinar-to-ductal metaplasia (ADM) formation, and carcinogenesis [72]. In Pdx1-Cre; lox-stop-lox-Kras ${ }^{\mathrm{G} 12 \mathrm{D} /+}$; Ink $4 a^{l o x /+}$ murine pancreatic cancer and in pancreatic cancer patient specimens, Prrx1 is expressed in CAFs. High expression levels of Prrx1 in CAFs are associated with squamous subtypes of pancreatic cancer [64]. Patients with the squamous subtype have shorter survival (Median survival 30.0/23.7/25.6 months for abnormally differentiated endocrine exocrine, pancreatic progenitor, immunogenic subtypes, 13.3 months for the squamous subtype) [73]. Prrx1-deficient CAFs display a myCAF identity with increased expression of VIM, $\alpha$-SMA, SPARC, and COL1A1 due to attenuated plasticity. Targeting Prrx1 can, therefore, be a treatment strategy by reducing CAF plasticity by forcing myCAF differentiation and the conversion of tumor-promoting to tumor-restraining CAFs (Figure 1) [64].

\section{Cancer-Restraining Cancer-Associated Fibroblasts}

It has been demonstrated that static normal fibroblasts suppress polyoma virus-transformed tumor cells [74], suggesting that an innate function of fibroblasts is to protect against tumorigenesis [75]. Subtypes of tumor-restraining CAFs and their specific marker proteins have not been well characterized. A recent study suggests that a CAF subtype expresses Meflin, a glycosylphosphatidylinositol-anchored protein encoded by the immunoglobulin superfamily containing a leucine-rich repeat gene (ISLR) [76]. Meflin has been considered as an MSC marker and expressed in MSCs, fibroblasts, pericytes, perivascular cells, and bone marrow [77]. Meflin expression in CAFs correlates with longer overall survival of patients with pancreatic cancer. Tumors in KPC mice with Meflin deficiency are larger and more proliferative than those developed in KPC mice with intact Meflin. Meflin knockout KPC mice show shorter overall survival than Meflin wild-type KPC mice. Meflin suppresses poor differentiation of pancreatic cancer [76]. Meflin is expressed by approximately $40 \%$ of FAP-positive CAFs and by the majority of CAFs expressing GLI1 [76]. As mentioned above, GLI1 accelerates KRAS-initiated pancreatic tumorigenesis in Gli1 knock-in mice, suggesting that CAF heterogeneity may exist within GLI-positive fibroblasts (see Section 3).

CD271, also known as a neurotrophin receptor, nerve growth factor receptor, NGFR, or p75NTR, has been implicated in tumor growth [78]. CD271 has been identified as a marker of MSCs [79]. Expression of CD271 is also observed in hepatic and pancreatic stellate cells [80,81]. Histologically, CD271-positive CAFs/PSCs are observed on the edge rather than in the center of tumors [78]. Another study also shows that CD271 expression is higher in peripheral CAFs than in juxta-tumoral 
CAFs [82]. High stromal expression of CD271 is associated with longer overall survival of pancreatic cancer patients [78]. Intriguingly, CD271 is highly expressed in CAFs in regressive stromal compartments in neoadjuvant FOLFILINOX (folinic acid, 5-fluorouracil, irinotecan and oxaliplatin) treated pancreatic cancer patients [82]. Whether we can consider CD271 as a cancer-restraining CAF marker or not, and whether neoadjuvant chemotherapy can trigger the conversion of tumor-promoting to tumor-restraining CAFs or not, still needs to be clarified.

$\alpha$-SMA has been considered as a candidate marker of tumor-restraining CAFs based on the observation in a pancreatic cancer mouse model [13] (see Section 1). However, several studies have shown that the number of $\alpha$-SMA-positive CAFs correlates with shorter overall survival in esophageal and pancreatic cancer patients $[83,84]$. Since CAFs are a heterogeneous population in the tumor microenvironment, it is plausible to consider that the "stromal switch" involves the conversion of tumor-restraining CAFs to tumor-promoting CAFs [41]. The "stromal switch" can be drivers of cellular plasticity, such as Prrx1. Furthermore, it is important to identify such "stromal switches," which would provide more specific therapies for pancreatic cancer patients. The specific markers of cancer-restraining CAFs need further identification and characterization.

\section{Conclusions}

CAFs represent very heterogeneous subpopulations, which can function in tumor-promoting as well as in a tumor-restraining manner. CAFs are derived in a wide variety of different cell types such as pancreatic stellate cells, bone marrow-derived, or adipose-derived mesenchymal stem cells, adipocytes, pericytes, monocytes, and endothelial cells, leading to and supporting CAF heterogeneity. The tumor microenvironment can also trigger the conversion from one CAF subtype to another CAF subtype. Further identification and characterization of CAF subtypes are required, but it is also important to understand the diversity of CAF subtypes in the tumor microenvironment and in spatial proximity to tumor cells. The refinement of knowledge on CAF heterogeneity, and further identification of factors triggering the conversion of tumor-promoting to tumor-restraining CAFs, would provide more specific and personalized therapies for pancreatic cancer patients in the future.

Author Contributions: Writing—original draft preparation, Y.S. Resources, J.H. Supervision, J.K. All authors have read and agreed to the published version of the manuscript.

Funding: This research received no external funding.

Conflicts of Interest: The authors declare no conflict of interest.

\section{References}

1. Bray, F.; Ferlay, J.; Soerjomataram, I.; Siegel, R.L.; Torre, L.A.; Jemal, A. Global cancer statistics 2018: GLOBOCAN estimates of incidence and mortality worldwide for 36 cancers in 185 countries. CA Cancer J. Clin. 2018, 68, 394-424. [CrossRef]

2. Rahib, L.; Smith, B.D.; Aizenberg, R.; Rosenzweig, A.B.; Fleshman, J.M.; Matrisian, L.M. Projecting cancer incidence and deaths to 2030: The unexpected burden of thyroid, liver, and pancreas cancers in the United States. Cancer Res. 2014, 74, 2913-2921. [CrossRef]

3. Siegel, R.L.; Miller, K.D.; Jemal, A. Cancer statistics, 2020. CA Cancer J. Clin. 2020, 70, 7-30. [CrossRef]

4. Cunningham, D.; Chau, I.; Stocken, D.D.; Valle, J.W.; Smith, D.; Steward, W.; Harper, P.G.; Dunn, J.; Tudur-Smith, C.; West, J.; et al. Phase III Randomized Comparison of Gemcitabine Versus Gemcitabine Plus Capecitabine in Patients with Advanced Pancreatic Cancer. J. Clin. Oncol. 2009, 27, 5513-5518. [CrossRef]

5. Conroy, T.; Desseigne, F.; Ychou, M.; Bouché, O.; Guimbaud, R.; Bécouarn, Y.; Adenis, A.; Raoul, J.-L.; Gourgou-Bourgade, S.; De La Fouchardière, C.; et al. FOLFIRINOX versus Gemcitabine for Metastatic Pancreatic Cancer. N. Engl. J. Med. 2011, 364, 1817-1825. [CrossRef]

6. Von Hoff, D.D.; Ervin, T.; Arena, F.P.; Chiorean, E.G.; Infante, J.; Moore, M.; Seay, T.; Tjulandin, S.A.; Ma, W.W.; Saleh, M.N.; et al. Increased Survival in Pancreatic Cancer with nab-Paclitaxel plus Gemcitabine. N. Engl. J. Med. 2013, 369, 1691-1703. [CrossRef] 
7. Neoptolemos, J.P.; Palmer, D.H.; Ghaneh, P.; Psarelli, E.E.; Valle, J.W.; Halloran, C.M.; Faluyi, O.; O’Reilly, D.A.; Cunningham, D.; Wadsley, J.; et al. Comparison of adjuvant gemcitabine and capecitabine with gemcitabine monotherapy in patients with resected pancreatic cancer (ESPAC-4): A multicentre, open-label, randomised, phase 3 trial. Lancet 2017, 389, 1011-1024. [CrossRef]

8. Conroy, T.; Hammel, P.; Hebbar, M.; Ben Abdelghani, M.; Wei, A.C.; Raoul, J.-L.; Choné, L.; Francois, E.; Artru, P.; Biagi, J.J.; et al. FOLFIRINOX or Gemcitabine as Adjuvant Therapy for Pancreatic Cancer. N. Engl. J. Med. 2018, 379, 2395-2406. [CrossRef] [PubMed]

9. Kleeff, J.; Korc, M.; Apte, M.; La Vecchia, C.; Johnson, C.D.; Biankin, A.V.; Neale, R.E.; Tempero, M.; Tuveson, D.A.; Hruban, R.H.; et al. Pancreatic cancer. Nat. Rev. Dis. Prim. 2016, 2, 16022. [CrossRef] [PubMed]

10. Ho, W.J.; Jaffee, E.M.; Zheng, L. The tumour microenvironment in pancreatic cancer-Clinical challenges and opportunities. Nat. Rev. Clin. Oncol. 2020, 17, 527-540. [CrossRef]

11. Erkan, M.; Reiser-Erkan, C.; Michalski, C.W.; Deucker, S.; Sauliunaite, D.; Streit, S.; Esposito, I.; Friess, H.; Kleeff, J. Cancer-Stellate Cell Interactions Perpetuate the Hypoxia-Fibrosis Cycle in Pancreatic Ductal Adenocarcinoma. Neoplasia 2009, 11, 497-508. [CrossRef] [PubMed]

12. Sunami, Y.; Rebelo, A.; Kleeff, J. Lipid Metabolism and Lipid Droplets in Pancreatic Cancer and Stellate Cells. Cancers 2017, 10, 3. [CrossRef] [PubMed]

13. Özdemir, B.C.; Pentcheva-Hoang, T.; Carstens, J.L.; Zheng, X.; Wu, C.-C.; Simpson, T.R.; Laklai, H.; Sugimoto, H.; Kahlert, C.; Novitskiy, S.V.; et al. Depletion of Carcinoma-Associated Fibroblasts and Fibrosis Induces Immunosuppression and Accelerates Pancreas Cancer with Reduced Survival. Cancer Cell 2014, 25, 719-734. [CrossRef] [PubMed]

14. Hingorani, S.R.; Wang, L.; Multani, A.S.; Combs, C.; Deramaudt, T.B.; Hruban, R.H.; Rustgi, A.K.; Chang, S.; Tuveson, D.A. Trp53R172H and KrasG12D cooperate to promote chromosomal instability and widely metastatic pancreatic ductal adenocarcinoma in mice. Cancer Cell 2005, 7, 469-483. [CrossRef] [PubMed]

15. Yauch, R.L.; Gould, S.E.; Scales, S.J.; Tang, T.; Tian, H.; Ahn, C.P.; Marshall, D.; Fu, L.; Januario, T.; Kallop, D.; et al. A paracrine requirement for hedgehog signalling in cancer. Nat. Cell Biol. 2008, 455, 406-410. [CrossRef] [PubMed]

16. Bailey, J.M.; Swanson, B.J.; Hamada, T.; Eggers, J.P.; Singh, P.K.; Caffery, T.; Ouellette, M.M.; Hollingsworth, M.A. Sonic Hedgehog Promotes Desmoplasia in Pancreatic Cancer. Clin. Cancer Res. 2008, 14, 5995-6004. [CrossRef] [PubMed]

17. Olive, K.P.; Jacobetz, M.A.; Davidson, C.J.; Gopinathan, A.; McIntyre, D.; Honess, D.; Madhu, B.; Goldgraben, M.A.; Caldwell, M.E.; Allard, D.; et al. Inhibition of Hedgehog Signaling Enhances Delivery of Chemotherapy in a Mouse Model of Pancreatic Cancer. Science 2009, 324, 1457-1461. [CrossRef]

18. Rhim, A.D.; Oberstein, P.E.; Thomas, D.H.; Mirek, E.T.; Palermo, C.F.; Sastra, S.A.; Dekleva, E.N.; Saunders, T.; Becerra, C.P.; Tattersall, I.W.; et al. Stromal Elements Act to Restrain, Rather Than Support, Pancreatic Ductal Adenocarcinoma. Cancer Cell 2014, 25, 735-747. [CrossRef]

19. Infinity Reports Update from Phase 2 Study of Saridegib Plus Gemcitabine in Patients with Metastatic Pancreatic Cancer. Available online: https://www.businesswire.com/news/home/20120127005146/en/InfinityReports-Update-Phase-2-Study-Saridegib (accessed on 24 November 2020).

20. Pereira, B.A.; Vennin, C.; Papanicolaou, M.; Chambers, C.R.; Herrmann, D.; Morton, J.P.; Cox, T.R.; Timpson, P. CAF Subpopulations: A New Reservoir of Stromal Targets in Pancreatic Cancer. Trends Cancer 2019, 5, 724-741. [CrossRef]

21. Sun, Q.; Zhang, B.; Hu, Q.; Qin, Y.; Xu, W.; Liu, W.; Yu, X.-J.; Xu, J. The impact of cancer-associated fibroblasts on major hallmarks of pancreatic cancer. Theranostics 2018, 8, 5072-5087. [CrossRef]

22. Erkan, M.; Adler, G.; Apte, M.V.; Bachem, M.G.; Buchholz, M.; Detlefsen, S.; Esposito, I.; Friess, H.; Gress, T.M.; Habisch, H.J.; et al. StellaTUM: Current consensus and discussion on pancreatic stellate cell research. Gut 2012, 61, 172-178. [CrossRef] [PubMed]

23. Neuzillet, C.; Tijeras-Raballand, A.; Ragulan, C.; Cros, J.; Patil, Y.; Martinet, M.; Erkan, M.; Kleeff, J.; Wilson, J.; Apte, M.; et al. Inter- and intra-tumoural heterogeneity in cancer-associated fibroblasts of human pancreatic ductal adenocarcinoma. J. Pathol. 2019, 248, 51-65. [CrossRef] [PubMed]

24. Biffi, G.; Tuveson, D.A. Diversity and Biology of Cancer-Associated Fibroblasts. Physiol. Rev. 2020, 101, 147-176. [CrossRef] [PubMed] 
25. Huang, X.; He, C.; Hua, X.; Kan, A.; Mao, Y.; Sun, S.; Duan, F.; Wang, J.; Huang, P.; Li, S. Oxidative stress induces monocyte-to-myofibroblast transdifferentiation through p38 in pancreatic ductal adenocarcinoma. Clin. Transl. Med. 2020, 10, e41. [CrossRef] [PubMed]

26. Miyazaki, Y.; Oda, T.; Mori, N.; Kida, Y.S. Adipose-derived mesenchymal stem cells differentiate into pancreatic cancer-associated fibroblasts in vitro. FEBS Open Bio 2020, 10, 2268-2281. [CrossRef] [PubMed]

27. Sahai, E.; Astsaturov, I.; Cukierman, E.; DeNardo, D.G.; Egeblad, M.; Evans, R.M.; Fearon, D.; Greten, F.R.; Hingorani, S.R.; Hunter, T.; et al. A framework for advancing our understanding of cancer-associated fibroblasts. Nat. Rev. Cancer 2020, 20, 174-186. [CrossRef] [PubMed]

28. Kalluri, R. The biology and function of fibroblasts in cancer. Nat. Rev. Cancer 2016, 16, 582-598. [CrossRef]

29. Endo, S.; Nakata, K.; Ohuchida, K.; Takesue, S.; Nakayama, H.; Abe, T.; Koikawa, K.; Okumura, T.; Sada, M.; Horioka, K.; et al. Autophagy Is Required for Activation of Pancreatic Stellate Cells, Associated with Pancreatic Cancer Progression and Promotes Growth of Pancreatic Tumors in Mice. Gastroenterology 2017, 152, 1492-1506.e24. [CrossRef]

30. Sousa, C.M.; Biancur, D.E.; Wang, X.; Halbrook, C.J.; Sherman, M.H.; Zhang, L.; Kremer, D.; Hwang, R.F.; Witkiewicz, A.K.; Ying, H.; et al. Pancreatic stellate cells support tumour metabolism through autophagic alanine secretion. Nat. Cell Biol. 2016, 536, 479-483. [CrossRef]

31. Tape, C.J.; Ling, S.; Dimitriadi, M.; McMahon, K.M.; Worboys, J.D.; Leong, H.S.; Norrie, I.C.; Miller, C.J.; Poulogiannis, G.; Lauffenburger, D.A.; et al. Oncogenic KRAS Regulates Tumor Cell Signaling via Stromal Reciprocation. Cell 2016, 165, 910-920. [CrossRef]

32. Quante, M.; Tu, S.P.; Tomita, H.; Gonda, T.; Wang, S.S.; Takashi, S.; Baik, G.H.; Shibata, W.; DiPrete, B.; Betz, K.S.; et al. Bone Marrow-Derived Myofibroblasts Contribute to the Mesenchymal Stem Cell Niche and Promote Tumor Growth. Cancer Cell 2011, 19, 257-272. [CrossRef] [PubMed]

33. Karnoub, A.E.; Dash, A.B.; Vo, A.P.; Sullivan, A.; Brooks, M.W.; Bell, G.W.; Richardson, A.L.; Polyak, K.; Tubo, R.; Weinberg, R.A. Mesenchymal stem cells within tumour stroma promote breast cancer metastasis. Nat. Cell Biol. 2007, 449, 557-563. [CrossRef] [PubMed]

34. Waghray, M.; Yalamanchili, M.; Dziubinski, M.; Zeinali, M.; Erkkinen, M.; Yang, H.; Schradle, K.A.; Urs, S.; Di Magliano, M.P.; Welling, T.H.; et al. GM-CSF Mediates Mesenchymal-Epithelial Cross-talk in Pancreatic Cancer. Cancer Discov. 2016, 6, 886-899. [CrossRef] [PubMed]

35. Strong, A.L.; Pei, D.T.; Hurst, C.G.; Gimble, J.M.; Burow, M.E.; Bunnell, B.A. Obesity Enhances the Conversion of Adipose-Derived Stromal/Stem Cells into Carcinoma-Associated Fibroblast Leading to Cancer Cell Proliferation and Progression to an Invasive Phenotype. Stem Cells Int. 2017, 2017, 9216502. [CrossRef]

36. Öhlund, D.; Handly-Santana, A.; Biffi, G.; Elyada, E.; Almeida, A.S.; Ponz-Sarvise, M.; Corbo, V.; Oni, T.E.; Hearn, S.A.; Lee, E.J.; et al. Distinct populations of inflammatory fibroblasts and myofibroblasts in pancreatic cancer. J. Exp. Med. 2017, 214, 579-596. [CrossRef]

37. Biffi, G.; Oni, T.E.; Spielman, B.; Hao, Y.; Elyada, E.; Park, Y.; Preall, J.; Tuveson, D.A. IL1-Induced JAK/STAT Signaling Is Antagonized by TGF $\beta$ to Shape CAF Heterogeneity in Pancreatic Ductal Adenocarcinoma. Cancer Discov. 2019, 9, 282-301. [CrossRef]

38. Raz, Y.; Cohen, N.; Shani, O.; Bell, R.E.; Novitskiy, S.V.; Abramovitz, L.; Levy, C.; Milyavsky, M.; Leider-Trejo, L.; Moses, H.L.; et al. Bone marrow-derived fibroblasts are a functionally distinct stromal cell population in breast cancer. J. Exp. Med. 2018, 215, 3075-3093. [CrossRef]

39. Mohr, A.; Zwacka, R.M. The future of mesenchymal stem cell-based therapeutic approaches for cancer From cells to ghosts. Cancer Lett. 2018, 414, 239-249. [CrossRef]

40. Niess, H.; Einem, J.C.; Thomas, M.N.; Michl, M.; Angele, M.; Huss, R.; Günther, C.; Nelson, P.J.; Bruns, C.J.; Heinemann, V. Treatment of advanced gastrointestinal tumors with genetically modified autologous mesenchymal stromal cells (TREAT-ME1): Study protocol of a phase I/II clinical trial. BMC Cancer 2015, 15, 237. [CrossRef]

41. Miyai, Y.; Esaki, N.; Takahashi, M.; Enomoto, A. Cancer-associated fibroblasts that restrain cancer progression: Hypotheses and perspectives. Cancer Sci. 2020, 111, 1047-1057. [CrossRef]

42. Bochet, L.; Lehuédé, C.; Dauvillier, S.; Wang, Y.Y.; Dirat, B.; Laurent, V.; Dray, C.; Guiet, R.; Maridonneau-Parini, I.; Le Gonidec, S.; et al. Adipocyte-Derived Fibroblasts Promote Tumor Progression and Contribute to the Desmoplastic Reaction in Breast Cancer. Cancer Res. 2013, 73, 5657-5668. [CrossRef] [PubMed] 
43. Tang, H.; Chu, Y.; Huang, Z.; Cai, J.; Wang, Z. The metastatic phenotype shift toward myofibroblast of adipose-derived mesenchymal stem cells promotes ovarian cancer progression. Carcinogenesis 2020, 41, 182-193. [CrossRef] [PubMed]

44. Byrnes, L.E.; Wong, D.M.; Subramaniam, M.; Meyer, N.P.; Gilchrist, C.L.; Knox, S.M.; Tward, A.D.; Ye, C.J.; Sneddon, J.B. Lineage dynamics of murine pancreatic development at single-cell resolution. Nat. Commun. 2018, 9, 3922. [CrossRef] [PubMed]

45. Garcia, P.E.; Adoumie, M.; Kim, E.C.; Zhang, Y.; Scales, M.K.; El-Tawil, Y.S.; Shaikh, A.Z.; Wen, H.-J.; Bednar, F.; Allen, B.L.; et al. Differential Contribution of Pancreatic Fibroblast Subsets to the Pancreatic Cancer Stroma. Cell. Mol. Gastroenterol. Hepatol. 2020, 10, 581-599. [CrossRef]

46. Barakat, M.T.; Humke, E.W.; Scott, M.P. Learning from Jekyll to control Hyde: Hedgehog signaling in development and cancer. Trends Mol. Med. 2010, 16, 337-348. [CrossRef]

47. Mathew, E.; Collins, M.A.; Fernandez-Barrena, M.G.; Holtz, A.M.; Yan, W.; Hogan, J.O.; Tata, Z.; Allen, B.L.; Fernandez-Zapico, M.E.; Di Magliano, M.P. The Transcription Factor GLI1 Modulates the Inflammatory Response during Pancreatic Tissue Remodeling. J. Biol. Chem. 2014, 289, 27727-27743. [CrossRef]

48. Rajurkar, M.; De Jesus-Monge, W.E.; Driscoll, D.R.; Appleman, V.A.; Huang, H.; Cotton, J.L.; Klimstra, D.S.; Zhu, L.J.; Simin, K.; Xu, L.; et al. The activity of Gli transcription factors is essential for Kras-induced pancreatic tumorigenesis. Proc. Natl. Acad. Sci. USA 2012, 109, E1038-E1047. [CrossRef]

49. Larsen, B.M.; Hrycaj, S.M.; Newman, M.; Li, Y.; Wellik, D.M. MesenchymalHox6function is required for mouse pancreatic endocrine cell differentiation. Development 2015, 142, 3859-3868. [CrossRef]

50. Österreicher, C.H.; Penz-Österreicher, M.; Grivennikov, S.I.; Guma, M.; Koltsova, E.K.; Datz, C.; Sasik, R.; Hardiman, G.; Karin, M.; Brenner, D.A. Fibroblast-specific protein 1 identifies an inflammatory subpopulation of macrophages in the liver. Proc. Natl. Acad. Sci. USA 2011, 108, 308-313. [CrossRef]

51. Li, H.; Courtois, E.T.; Sengupta, D.; Tan, Y.; Chen, K.H.; Goh, J.J.L.; Kong, S.L.; Chua, C.; Hon, L.K.; Tan, W.S.; et al. Reference component analysis of single-cell transcriptomes elucidates cellular heterogeneity in human colorectal tumors. Nat. Genet. 2017, 49, 708-718. [CrossRef]

52. Hofheinz, R.-D.; Al-Batran, S.-E.; Hartmann, F.; Hartung, G.; Jäger, D.; Renner, C.; Tanswell, P.; Kunz, U.; Amelsberg, A.; Kuthan, H.; et al. Stromal Antigen Targeting by a Humanised Monoclonal Antibody: An Early Phase II Trial of Sibrotuzumab in Patients with Metastatic Colorectal Cancer. Oncol. Res. Treat. 2003, 26, 44-48. [CrossRef] [PubMed]

53. Surowiak, P.; Murawa, D.; Materna, V.; Maciejczyk, A.; Pudelko, M.; Ciesla, S.; Breborowicz, J.; Murawa, P.; Zabel, M.; Dietel, M.; et al. Occurence of stromal myofibroblasts in the invasive ductal breast cancer tissue is an unfavourable prognostic factor. Anticancer Res. 2007, 27, 2917-2924.

54. Tsujino, T.; Seshimo, I.; Yamamoto, H.; Ngan, C.Y.; Ezumi, K.; Takemasa, I.; Ikeda, M.; Sekimoto, M.; Matsuura, N.; Monden, M. Stromal Myofibroblasts Predict Disease Recurrence for Colorectal Cancer. Clin. Cancer Res. 2007, 13, 2082-2090. [CrossRef] [PubMed]

55. Heldin, C.-H. Targeting the PDGF signaling pathway in tumor treatment. Cell Commun. Signal. 2013, 11, 97. [CrossRef] [PubMed]

56. Nurmik, M.; Ullmann, P.; Rodriguez, F.; Haan, S.; Letellier, E. In search of definitions: Cancer-associated fibroblasts and their markers. Int. J. Cancer 2020, 146, 895-905. [CrossRef] [PubMed]

57. Shindo, K.; Aishima, S.; Ohuchida, K.; Fujiwara, K.; Fujino, M.; Mizuuchi, Y.; Hattori, M.; Mizumoto, K.; Tanaka, M.; Oda, Y. Podoplanin expression in cancer-associated fibroblasts enhances tumor progression of invasive ductal carcinoma of the pancreas. Mol. Cancer 2013, 12, 168. [CrossRef] [PubMed]

58. Kobayashi, H.; Enomoto, A.; Woods, S.L.; Burt, A.D.; Takahashi, M.; Worthley, D.L. Cancer-associated fibroblasts in gastrointestinal cancer. Nat. Rev. Gastroenterol. Hepatol. 2019, 16, 282-295. [CrossRef]

59. Sulzmaier, F.J.; Jean, C.; Schlaepfer, D.D. FAK in cancer: Mechanistic findings and clinical applications. Nat. Rev. Cancer 2014, 14, 598-610. [CrossRef]

60. Zaghdoudi, S.; Decaup, E.; Belhabib, I.; Samain, R.; Cassant-Sourdy, S.; Rochotte, J.; Brunel, A.; Schlaepfer, D.; Cros, J.; Neuzillet, C.; et al. FAK activity in cancer-associated fibroblasts is a prognostic marker and a druggable key metastatic player in pancreatic cancer. EMBO Mol. Med. 2020, 12, e12010. [CrossRef]

61. Elyada, E.; Bolisetty, M.; Laise, P.; Flynn, W.F.; Courtois, E.T.; Burkhart, R.A.; Teinor, J.A.; Belleau, P.; Biffi, G.; Lucito, M.S.; et al. Cross-Species Single-Cell Analysis of Pancreatic Ductal Adenocarcinoma Reveals Antigen-Presenting Cancer-Associated Fibroblasts. Cancer Discov. 2019, 9, 1102-1123. [CrossRef] 
62. Hosein, A.N.; Huang, H.; Wang, Z.; Parmar, K.; Du, W.; Huang, J.; Maitra, A.; Olson, E.N.; Verma, U.; Brekken, R. Cellular heterogeneity during mouse pancreatic ductal adenocarcinoma progression at single-cell resolution. JCI Insight 2019, 23, e129212. [CrossRef] [PubMed]

63. Dominguez, C.X.; Müller, S.; Keerthivasan, S.; Koeppen, H.; Hung, J.; Gierke, S.; Breart, B.; Foreman, O.; Bainbridge, T.W.; Castiglioni, A.; et al. Single-Cell RNA Sequencing Reveals Stromal Evolution into LRRC15+ Myofibroblasts as a Determinant of Patient Response to Cancer Immunotherapy. Cancer Discov. 2019, 10, 232-253. [CrossRef]

64. Feldmann, K.; Maurer, C.; Peschke, K.; Teller, S.; Schuck, K.; Steiger, K.; Engleitner, T.; Öllinger, R.; Nomura, A.; Wirges, N.; et al. Mesenchymal Plasticity Regulated by Prrx1 Drives Aggressive Pancreatic Cancer Biology. Gastroenterology 2020, 7, 35147. [CrossRef]

65. Bernard, V.; Semaan, A.; Huang, J.; Lucas, F.A.S.; Mulu, F.C.; Stephens, B.M.; Guerrero, P.A.; Huang, Y.; Zhao, J.; Kamyabi, N.; et al. Single-Cell Transcriptomics of Pancreatic Cancer Precursors Demonstrates Epithelial and Microenvironmental Heterogeneity as an Early Event in Neoplastic Progression. Clin. Cancer Res. 2019, 25, 2194-2205. [CrossRef] [PubMed]

66. Kudo, A. Introductory review: Periostin-Gene and protein structure. Cell. Mol. Life Sci. 2017, 74, 4259-4268. [CrossRef] [PubMed]

67. Excoffon, K.J.; Avenarius, M.R.; Hansen, M.R.; Kimberling, W.J.; Najmabadi, H.; Smith, R.J.H.; Zabner, J. The Coxsackievirus and Adenovirus Receptor: A new adhesion protein in cochlear development. Hear. Res. 2006, 215, 1-9. [CrossRef] [PubMed]

68. Mankoo, B.S.; Skuntz, S.; Harrigan, I.; Grigorieva, E.; Candia, A.; Wright, C.V.E.; Arnheiter, H.; Pachnis, V. The concerted action of Meox homeobox genes is required upstream of genetic pathways essential for the formation, patterning and differentiation of somites. Development 2003, 130, 4655-4664. [CrossRef]

69. Morgan, A.; Koboldt, D.C.; Barrie, E.S.; Crist, E.R.; García, G.G.; Mezzavilla, M.; Faletra, F.; Mosher, T.M.; Wilson, R.K.; Blanchet, C.; et al. Mutations in PLS1, encoding fimbrin, cause autosomal dominant nonsyndromic hearing loss. Hum. Mutat. 2019, 40, 2286-2295. [CrossRef]

70. Maitra, A.; Hruban, R.H. Pancreatic Cancer. Annu. Rev. Pathol. Mech. Dis. 2008, 3, 157-188. [CrossRef]

71. Makohon-Moore, A.; Iacobuzio-Donahue, C.A. Pancreatic cancer biology and genetics from an evolutionary perspective. Nat. Rev. Cancer 2016, 16, 553-565. [CrossRef]

72. Reichert, M.; Takano, S.; Von Burstin, J.; Kim, S.-B.; Lee, J.-S.; Ihida-Stansbury, K.; Hahn, C.; Heeg, S.; Schneider, G.; Rhim, A.D.; et al. The Prrx1 homeodomain transcription factor plays a central role in pancreatic regeneration and carcinogenesis. Genes Dev. 2013, 27, 288-300. [CrossRef] [PubMed]

73. Bailey, P.; Chang, D.K.; Nones, K.; Johns, A.L.; Patch, A.M.; Gingras, M.C.; Miller, D.K.; Christ, A.N.; Bruxner, T.J.; Quinn, M.C.; et al. Genomic analyses identify molecular subtypes of pancreatic cancer. Nature 2016, 531, 47-52. [CrossRef] [PubMed]

74. Stoker, M.G.; Shearer, M.; O'Neill, C. Growth inhibition of polyoma-transformed cells by contact with static normal fibroblasts. J. Cell Sci. 1966, 1, 297-310. [PubMed]

75. Klein, G. Evolutionary aspects of cancer resistance. Semin. Cancer Biol. 2014, 25, 10-14. [CrossRef]

76. Mizutani, Y.; Kobayashi, H.; Iida, T.; Asai, N.; Masamune, A.; Hara, A.; Esaki, N.; Ushida, K.; Mii, S.; Shiraki, Y.; et al. Meflin-Positive Cancer-Associated Fibroblasts Inhibit Pancreatic Carcinogenesis. Cancer Res. 2019, 79, 5367-5381. [CrossRef]

77. Maeda, K.; Enomoto, A.; Hara, A.; Asai, N.; Kobayashi, T.; Horinouchi, A.; Maruyama, S.; Ishikawa, Y.; Nishiyama, T.; Kiyoi, H.; et al. Identification of Meflin as a Potential Marker for Mesenchymal Stromal Cells. Sci. Rep. 2016, 6, 22288. [CrossRef]

78. Fujiwara, K.; Ohuchida, K.; Mizumoto, K.; Shindo, K.; Eguchi, D.; Kozono, S.; Ikenaga, N.; Ohtsuka, T.; Takahata, S.; Aishima, S.; et al. CD271+ Subpopulation of Pancreatic Stellate Cells Correlates with Prognosis of Pancreatic Cancer and Is Regulated by Interaction with Cancer Cells. PLoS ONE 2012, 7, e52682. [CrossRef]

79. Bühring, H.-J.; Battula, V.L.; Treml, S.; Schewe, B.; Kanz, L.; Vogel, W. Novel Markers for the Prospective Isolation of Human MSC. Ann. N. Y. Acad. Sci. 2007, 1106, 262-271. [CrossRef]

80. Trim, N.; Morgan, S.; Evans, M.; Issa, R.; Fine, D.; Afford, S.; Wilkins, B.; Iredale, J.P. Hepatic Stellate Cells Express the Low Affinity Nerve Growth Factor Receptor p75 and Undergo Apoptosis in Response to Nerve Growth Factor Stimulation. Am. J. Pathol. 2000, 156, 1235-1243. [CrossRef] 
81. Haas, S.L.; Fitzner, B.; Jaster, R.; Wiercinska, E.; Gaitantzi, H.; Jesenowski, R.; Löhr, J.-M.; Singer, M.V.; Dooley, S.; Breitkopf, K. Transforming growth factor- $\beta$ induces nerve growth factor expression in pancreatic stellate cells by activation of the ALK-5 pathway. Growth Factors 2009, 27, 289-299. [CrossRef]

82. Nielsen, M.F.B.; Mortensen, M.B.; Sørensen, M.D.; Wirenfeldt, M.; Kristensen, B.W.; Schrøder, H.D.; Pfeiffer, P.; Detlefsen, S. Spatial and phenotypic characterization of pancreatic cancer-associated fibroblasts after neoadjuvant treatment. Histol. Histopathol. 2020, 35, 811-825. [CrossRef] [PubMed]

83. Underwood, T.J.; Hayden, A.L.; Derouet, M.; Garcia, E.; Noble, F.; White, M.J.; Thirdborough, S.; Mead, A.; Clemons, N.; Mellone, M.; et al. Cancer-associated fibroblasts predict poor outcome and promote periostin-dependent invasion in oesophageal adenocarcinoma. J. Pathol. 2015, 235, 466-477. [CrossRef] [PubMed]

84. Fujita, H.; Ohuchida, K.; Mizumoto, K.; Nakata, K.; Yu, J.; Kayashima, T.; Cui, L.; Manabe, T.; Ohtsuka, T.; Tanaka, M. $\alpha$-Smooth Muscle Actin Expressing Stroma Promotes an Aggressive Tumor Biology in Pancreatic Ductal Adenocarcinoma. Pancreas 2010, 39, 1254-1262. [CrossRef] [PubMed]

Publisher's Note: MDPI stays neutral with regard to jurisdictional claims in published maps and institutional affiliations.

(C) 2020 by the authors. Licensee MDPI, Basel, Switzerland. This article is an open access article distributed under the terms and conditions of the Creative Commons Attribution (CC BY) license (http://creativecommons.org/licenses/by/4.0/). 